to these variables monthly, communities can diagnose problems more precisely and identify improvements more quickly.

\section{EVALUATION OF THE EDMONTON ZONE TRIPLE AIM INITIATIVE: BUILDING AND IMPLEMENTING A MEASUREMENT SYSTEM FOR IMPROVEMENT WITH COMPLEX, VULNERABLE CLIENTS}

Eric VanSpronsen, Christine Vandenberghe, Melanie Hennig, Tristan Robinson, Lana Socha, SungHyun Kang, Xiaoming Wang, Lorraine Telford, Dorah Conteh. Alberta Health Services, Canada

\subsection{6/bmjoq-2017-|HI.26}

Background The Alberta Health Services, Edmonton Zone Triple Aim Initiative launched in January, 2013. The seven participating clinical teams target complex, vulnerable patients in inner-city Edmonton, Alberta, Canada. The Initiative follows the Institute for Healthcare Improvement's Better Health Lower Cost Road Map and pursues the Quadruple Aim. Grant funding was provided by MERCK Canada and Alberta Innovates.

Objectives The objectives of the evaluation were: 1) Determine the extent to which the Aims of the Initiative have been met; and 2) Build a measurement system to monitor the performance of the team quality improvement efforts.

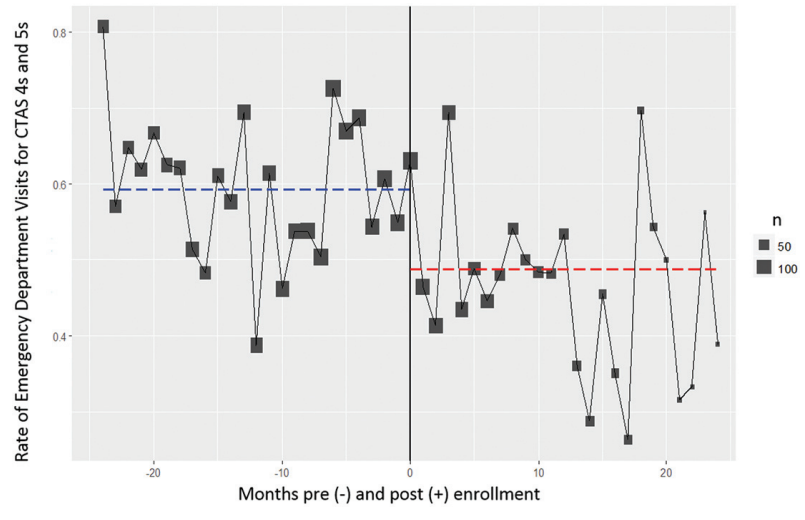

CTAS= Canadian Triage \& Acuity Scale (CTAS $4 \& 5$ are less acute)

Abstract 1033 Figure 1 Average monthly rates for emergency department visits for CTAS $4 \mathrm{~s}$ and $5 \mathrm{~s}$. CTAS=Canadian Triage and Acuity Scale (CTAS 4 and 5 are less acute)

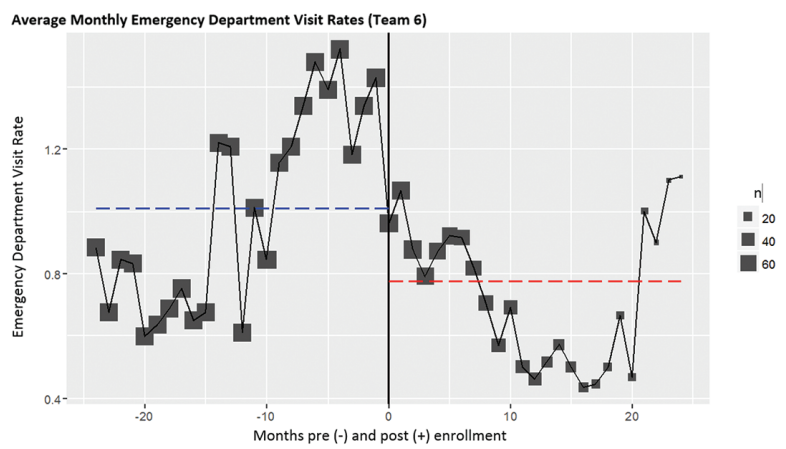

Abstract 1033 Figure 2 Average monthly emergency department visit rates (team 6)

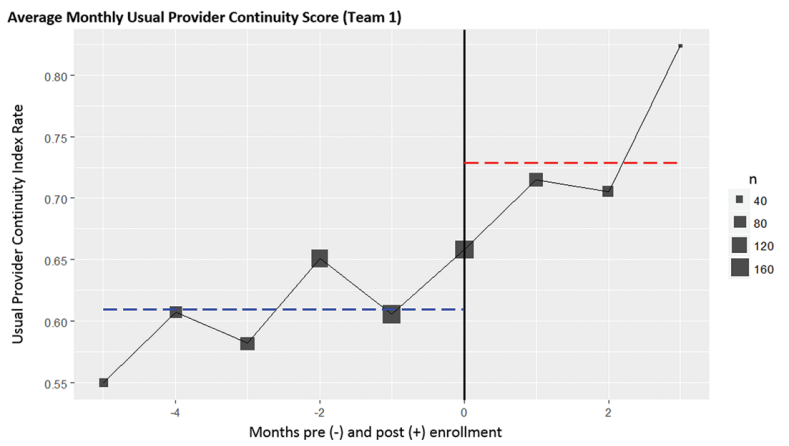

Abstract 1033 Figure 4 Average monthly usual provider continuity score (team 1)

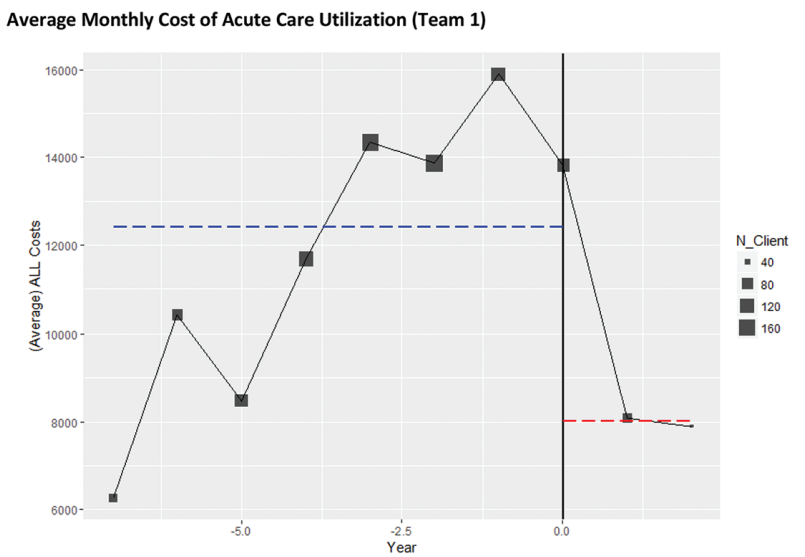

Abstract 1033 Figure 5 Average monthly cost of acute care utilisation (team 1)

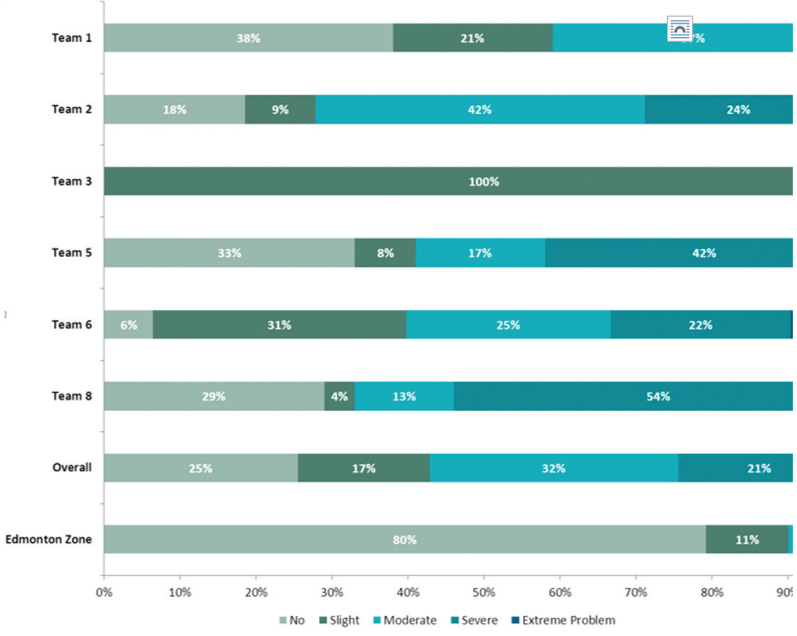

Abstract 1033 Figure 6 EQ-5D-5L Mobility dimension responses

Methods Over 40 providers and 445 patients participated. Data collection include: administrative data; patient surveys; and patient and staff interviews. Analyses of system level data (e.g., emergency department visits; inpatient stays; and 


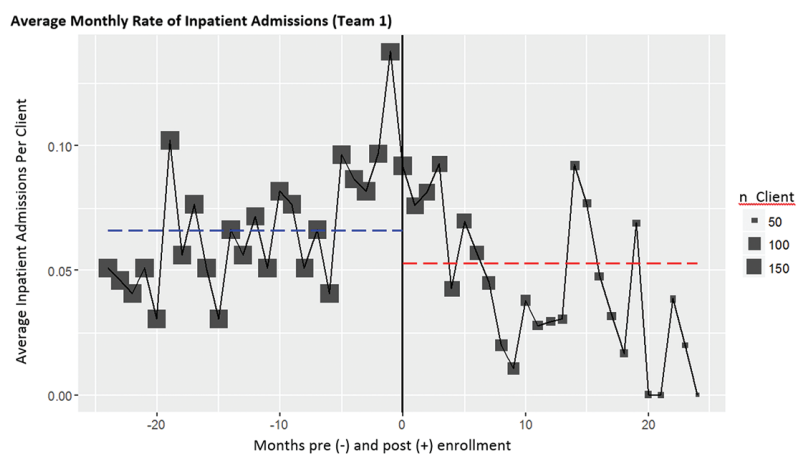

Abstract 1033 Figure 3 Average monthly rate of inpatient admissions (team 1)

physician continuity) included both descriptive and statistical modelling approaches from a pre/post comparison perspective. Results Across all teams there were strong improvements in self-reported experience for both patients and providers. Some teams demonstrated reduced acute care utilisation and cost, and higher continuity with a family physician. Better outcomes were linked with teams delivering on more elements of the Managing Complex Change model: having a vision, skills, incentives, adequate resources and an action plan.

Conclusions The evaluation demonstrates that the teams have improved care for their patients. Lessons learned from this evaluation will be critical for the Initiative moving forward, and also others working with similar populations. Recommendations from the evaluation for implementing system-level improvement initiatives will be discussed, as well as recommendation for implementing measurement systems with complex patients.

\section{THE EMERGENCY LAPAROTOMY COLLABORATIVE: SCALING UP AN IMPROVEMENT BUNDLE FOR HIGH RISK SURGICAL PATIENTS}

${ }^{1}$ Carol Peden, ${ }^{2}$ Geeta Aggarwal, ${ }^{2}$ Nial Quiney, ${ }^{3}$ Anne Pullyblank, ${ }^{4}$ Tim Stephens. ${ }^{1}$ Keck Medicine of USC, US; ${ }^{2}$ Royal Surrey Hospital NHS Trust, UK; ${ }^{3}$ North Bristol NHS Trust, UK; ${ }^{4}$ Queen Mary, University of London, UK

\subsection{6/bmjoq-2017-|HI.27}

Background Emergency intra-abdominal laparotomy is a common surgical procedure. Mortality is high with $11 \%-15 \%$ of patients dying within 30 days of surgery. Complication rates are also high and $>25 \%$ of patients remain in hospital for >20 days. A previous study, ELPQuIC, successfully used a care bundle to reduce mortality in four hospitals.

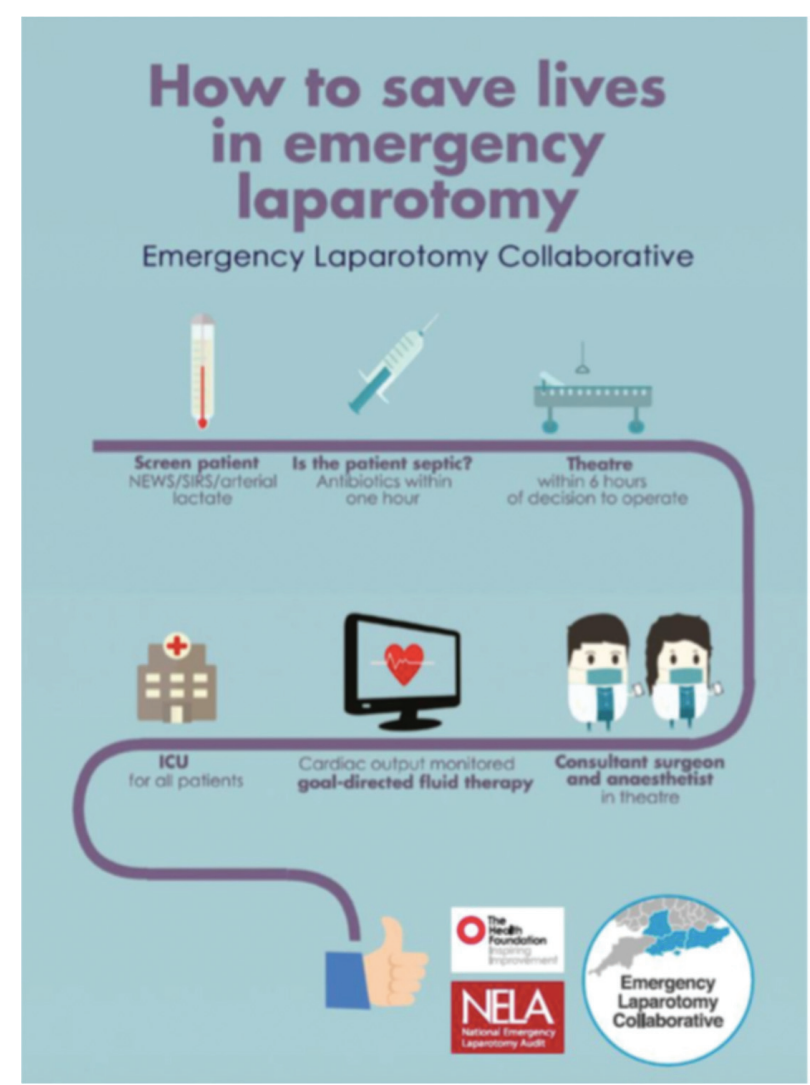

Abstract 1040 Figure 1 Diagram of the 'ELPQulC' Care Bundle

Objectives The aim of the Emergency Laparotomy Collaborative was to scale implementation of the ELPQuIC bundle to 24 NHS Trusts within three Academic Networks to reduce mortality, complications and length of stay.

Methods We used the IHI Breakthrough Series Collaborative Model to bring $100+$ staff together over two years with 5 large events and 4 local quality improvement events. Data collection was through the National Emergency Laparotomy Audit (NELA). Economic analysis was undertaken. Using NELA data we distributed comparative dashboards showing care bundle adherence and patient outcomes quarterly. The collaborative model enabled Trusts to share progress through dialogue, group reflection and celebration of success.

Results 5793 patients had an emergency laparotomy between October 2015 and December 2016. Crude mortality decreased from $9.8 \%$ to $8.7 \%$ and length of stay decreased by 1.3 days. There were significant improvements in delivery of care bundle components. Economic analysis showed potential savings of $£ 2 \mathrm{M}$ in 15 months, primarily through decreased length of

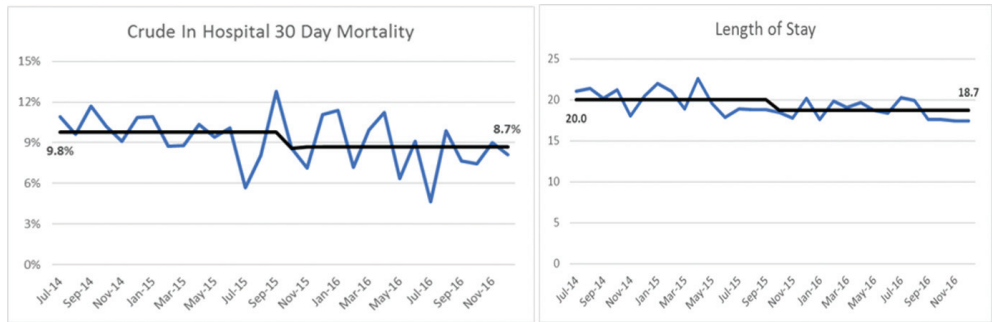

Abstract 1040 Figure 2 Run charts of mortality and length of stay from all hospitals. Intervention began October 2015. 